\title{
Heterogeneity in Li-Fraumeni families: p53 mutation analysis and immunohistochemical staining
}

\author{
C MacGeoch, G Turner, L G Bobrow, D M Barnes, D T Bishop, N K Spurr
}

\begin{abstract}
We have screened two families for constitutional TP53 mutations, one family with Li-Fraumeni syndrome and the other with features of this syndrome. We report a germline mutation in exon 7 of the TP53 gene in the family with " $\mathrm{Li}-F r a u m e n i-$ like" syndrome. The mutation occurred at codon 245 and causes a Gly-Ser amino acid change. It was inherited by both affected and unaffected subjects. Malignant tumours from all members of this family showed strong positive nuclear immunohistochemical staining with antibodies CM-1 and DO1, directed against TP53. In contrast, no constitutional TP53 mutations were found in a "classic" $\mathrm{Li}$ Fraumeni family. In this family positive staining was seen in both malignant and normal tissues. These results support previous findings that variants of the Li-Fraumeni syndrome exist since not all LFS families carry TP53 germline mutations. Secondly, immunohistochemical positivity is not synonymous with an underlying mutation and is therefore inadequate as an exclusive diagnostic marker.
\end{abstract}

(f Med Genet 1995;32:186-190)

Imperial Cancer Research Fund Human Genetic Resources, Clare Hall Laboratories, South Mimms, Potters Bar Merts EN6 3LD, UK C MacGeoch

N K Spur

Imperial Cancer Research Fund Genetic Epidemiology Laboratory, St James's

University Hospital,

Leeds LS9 7TF, UK

G Turner

D T Bishop

Imperial Cancer Research Fund Clinical Oncology Unit, Guy's Hospital, London SE1 9RT, UK

L G Bobrow

D M Barnes

Correspondence to: Dr MacGeoch.

Received 8 August 1994 Revised version accepted for publication 28 October 1994
Li-Fraumeni syndrome (LFS) is a devastating autosomal dominant disorder of multiple cancers that are difficult to manage and are often lethal. Individually, these cancers are clinically and histopathologically indistinguishable from their counterparts which arise in the general population. Approximately $50 \%$ of cancers in reported Li-Fraumeni families occur before 30 years of age. The "classic" Li-Fraumeni syndrome is described as the diagnosis of a sarcoma before the age of 45 years and at least two first degree relatives with cancer before 45 years. In young adults, premenopausal breast cancer is by far the most common neoplasm. The commonest childhood cancers are soft tissue sarcomas below 5 years of age and osteosarcomas in adolescence. Acute leukaemia, brain tumours, and carcinomas of the adrenal cortex are often observed in families with this syndrome. ${ }^{1}$

In 1990, five families with Li-Fraumeni syndrome were reported to show germline mutations in the TP53 gene. ${ }^{2}$ TP53 is generally classified as a tumour suppressor gene and is currently the commonest known site of somatic mutations in human cancers. Germline se- quence alterations in the TP53 gene have also been detected in families with breast cancer, sarcomas, and other forms of neoplasia, ${ }^{2}$ and in sporadic human tumours of the brain, lung, liver, oesophagus, and colon. ${ }^{3}$ Subsequent studies have shown that germline TP53 mutations occur in some "Li-Fraumeni families"4-6 but not others. ${ }^{78}$ All reported germline mutations in TP53 occur within the conserved regions of the gene with $50 \%$ of all mutations occuring in exon 7; no mutations have been published outside exons 4 to $9 .{ }^{9}$ Inherited TP53 mutations probably convey a substantial increase in cancer risk, and knowledge of the inherited change will have important implications for those family members at risk of having inherited the gene and for those offering genetic counselling to the families.

Early onset breast cancer commonly represented in LFS is the commonest fatal cancer of women in developed countries. Some families show evidence of a dominantly inherited susceptibility, often only with breast cancer but sometimes also with ovarian cancer. Among this latter group, almost all show linkage to a gene mapping to $17 \mathrm{q}, \mathrm{BRCA} 1,{ }^{10}$ as do approximately $40 \%$ of families with apparent susceptibility only to breast cancer. Several studies have shown that germline TP53 mutations occur rarely in hereditary early onset breast cancer families except in Li-Fraumeni syndrome in which it is a feature. ${ }^{1112}$

We have identified two families which clinically suggest the $\mathrm{Li}$-Fraumeni syndrome. Both families were analysed immunohistochemically for the presence of abnormally stabilised protein and for the presence of germline TP53 mutations.

\section{Materials and methods \\ FAMILIES}

As part of a project to ascertain premenopausal women with both a personal and family history of breast cancer, two families were identified with features of the Li-Fraumeni syndrome. Blood and tumour samples were obtained from the probands and from all the available family members referred to the ICRF Genetic Epidemiology Laboratory, St James's University Hospital, Leeds.

Family 1 fulfils the criteria of "classic" $\mathrm{Li}$ Fraumeni syndrome. The proband (I-2) had a liposarcoma at 13 years with a recurrence at the age of 18 and developed mammary carcinoma at the age of 40 . One of her children (II.3) had a parosteal sarcoma aged 18 and 
A

B

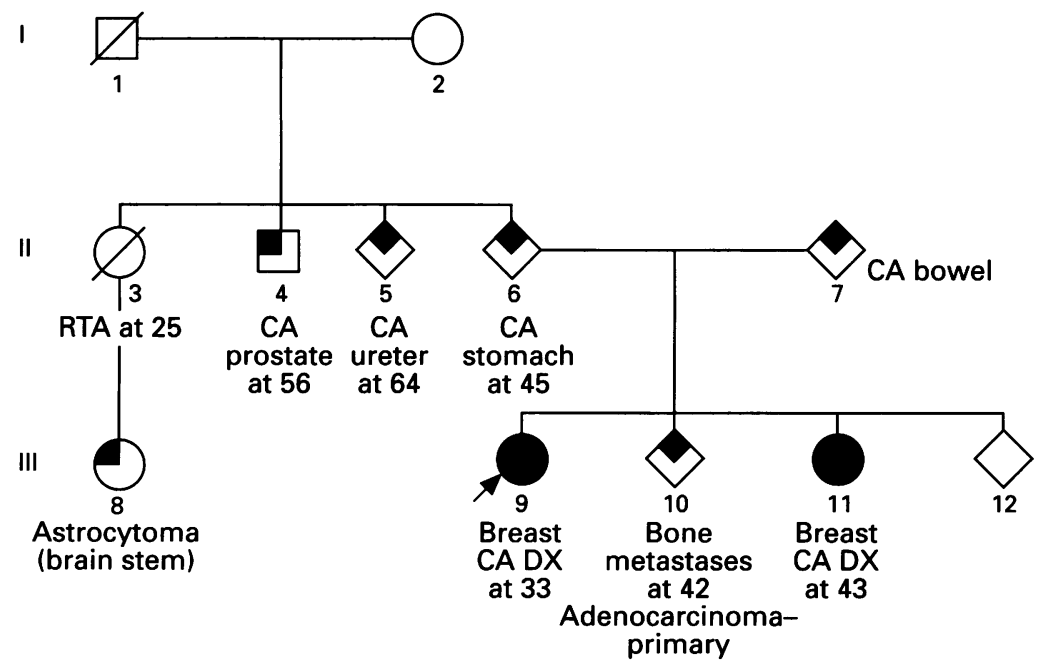

Figure 1 Pedigrees of two families with Li-Fraumeni syndrome. Panel A, family 1 fulfils the criteria of classic Li-Fraumeni syndrome. Panel B, family 2 exhibits "Li-Fraumenilike" series of tumours. carried out using the monoclonal antibody $\mathrm{DO}^{14}$ and polyclonal antibody $\mathrm{CM}-1$, using a peroxidase conjugated streptavidin biotin technique described in detail elsewhere. ${ }^{15}$ Specific brown staining of nuclei was taken as positive indication of the presence of an abnormal accumulation of stabilised p53 protein. Sections of breast tumour from other sources known to stain positively for p53 were used as a positive control and were included in each batch of staining. Primary antibody was substituted with phosphate buffered saline (PBS) in duplicate test sections for negative controls.

\section{Results}

PEDIGREES AND MUTATION ANALYSIS

The pedigrees of the two families are shown in fig 1, panels A and B. PCR amplification and direct sequencing of TP53 products captured on magnetic beads were used to identify germline mutations. Initially samples were analysed for mutations in exon 7 of the p53 gene where the majority of LFS mutations have been identified.

In family 1 no variant band was detected upon sequence analysis of this region. Further sequence analysis was undertaken in exons 5 to 9 of the TP53 gene. No mutations were detected in any of the blood samples analysed from this family.

In family 2, a germline mutation in the TP53 gene was detected. The pedigree and the sequence mutation data are shown in fig 2 . The proband of the family (III.9) had a mutation at codon 245 in exon 7 . Both the wild type and mutant alleles are present. The mutation causes a G-A transition at the first position (GGC-AGC) and results in a Gly-Ser amino acid change. The proband's affected sister who has breast cancer also carries the same mutated allele. An unaffected sib also has the mutation at codon 245. DNA was available from the parent who had bowel cancer but was found not to show the mutation. However, an affected family member with astrocytoma (III.8) on the other side of the family was shown to carry the mutation. This indicates that the mutant allele is inherited through this parental family line. Both the mutated AGC and the normal GGC TP53 alleles with respect to codon 245 were observed in all family members who carried the mutation.

\section{IMMUNOHISTOCHEMICAL ANALYSIS}

It was only possible to investigate three first degree relatives from family 1 . The fixed tissue of the proband $(I \cdot 2)$ exhibited positive staining with both antibodies in both malignant and benign epithelial cells. The two children who had developed tumours both had positive staining in many normal mesenchymal cells, particularly in the osteoblasts and endothelial cells as well as in the malignant tissues. An example of the staining pattern observed in $\mathrm{II} \cdot 4$ is shown in fig 3.

The staining patterns obtained using antiImmunohistochemical staining of available formalin fixed paraffin embedded tissue was body CMl in the carcinomas from people tested in family 2 is shown in fig 4 . Tissue from 


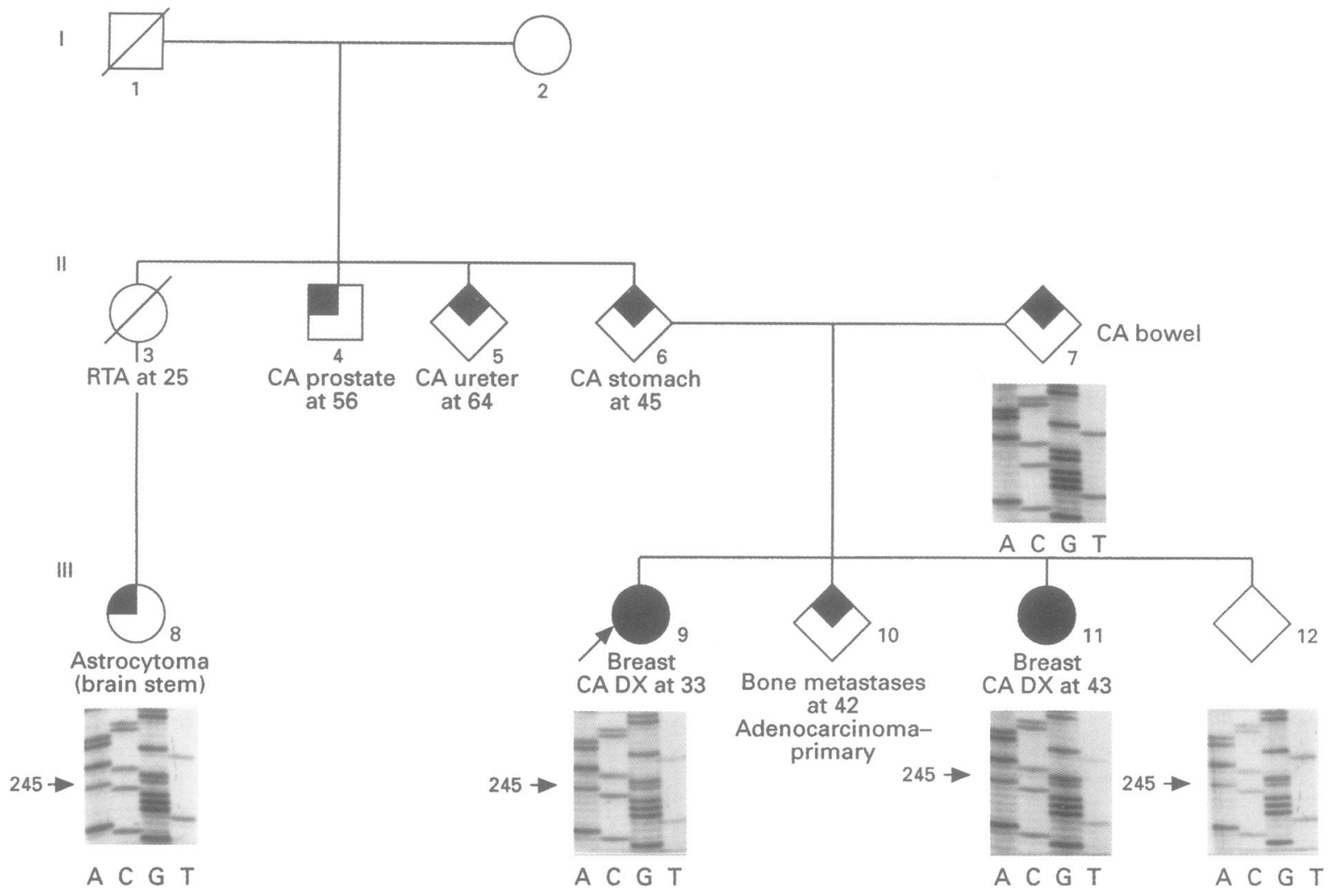

Figure 2 Position of TP53 gene mutation for family 2. Sequencing of the region spanning exons 5-8 was performed as described. The region in which the observed mutation occurred is shown. The boxed in segment from codons 242-248 identifies part of conserved region IV. The sequences are read from bottom to top. The arrow indicates position 245 at which the mutation occurred causing a Gly-Ser amino acid change. Expression of both the mutant and normal alleles can be seen in each person. Each sample was sequenced at least twice from separate PCR generated templates.
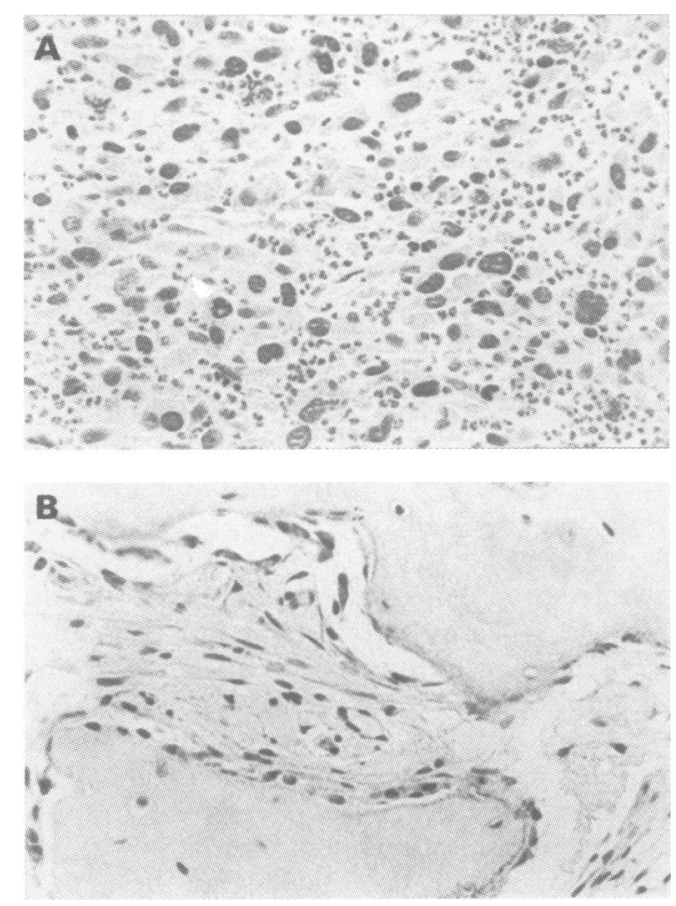

Figure 3 Immunohistochemical staining for p53 protein in $(A)$ malignant and $(B)$ normal tissue from $I I \cdot 4$ in family 1 who developed a parosteal sacroma at the age of 16. $(A)$ shows the majority of malignant cells staining positively for p53, (B) shows normal bone marrow with positively stained osteoblasts, fibroblasts, and endothelial cells. a ductal carcinoma in situ was available from the proband (III.9). More than $90 \%$ of the carcinoma cells showed strong nuclear staining for p53 protein. II 4 had an undifferentiated carcinoma of the prostrate which expressed prostatic specific antigen and showed p53 nuclear staining in over $75 \%$ of the malignant cells. Material containing undifferentiated carcinoma arising in the epithelium of the ureter was available from II $\cdot 5 ; 75 \%$ of the malignant cells expressed nuclear p53 protein. Family member II 6 had a gastric carcinoma with both well differentiated and poorly differentiated areas. Nuclear p53 expression was seen in more than $50 \%$ of the tumour cells in the well differentiated areas and was present in less than $10 \%$ of the tumour cells in poorly differentiated areas. Tumour material was not available from the brain stem astrocytoma in family member III.8. The metastatic adenocarcinoma arising within a lymph node from III- 10 showed positive staining for p53 protein in more than $75 \%$ of the tumour cells. Family member III- 11 had a grade III infiltrating ductal carcinoma of the breast in with 50 to $75 \%$ of the tumour cells showed nuclear expression of p53. In addition to the available malignant tissue there was always normal tissue available from all family members, sometimes in the same sample as the malignant tissue and sometimes in tissue from other organs. There was no evidence of staining of normal tissue from five of the six family members with either antibody. In the sixth (III.9) there were a few areas of weak 

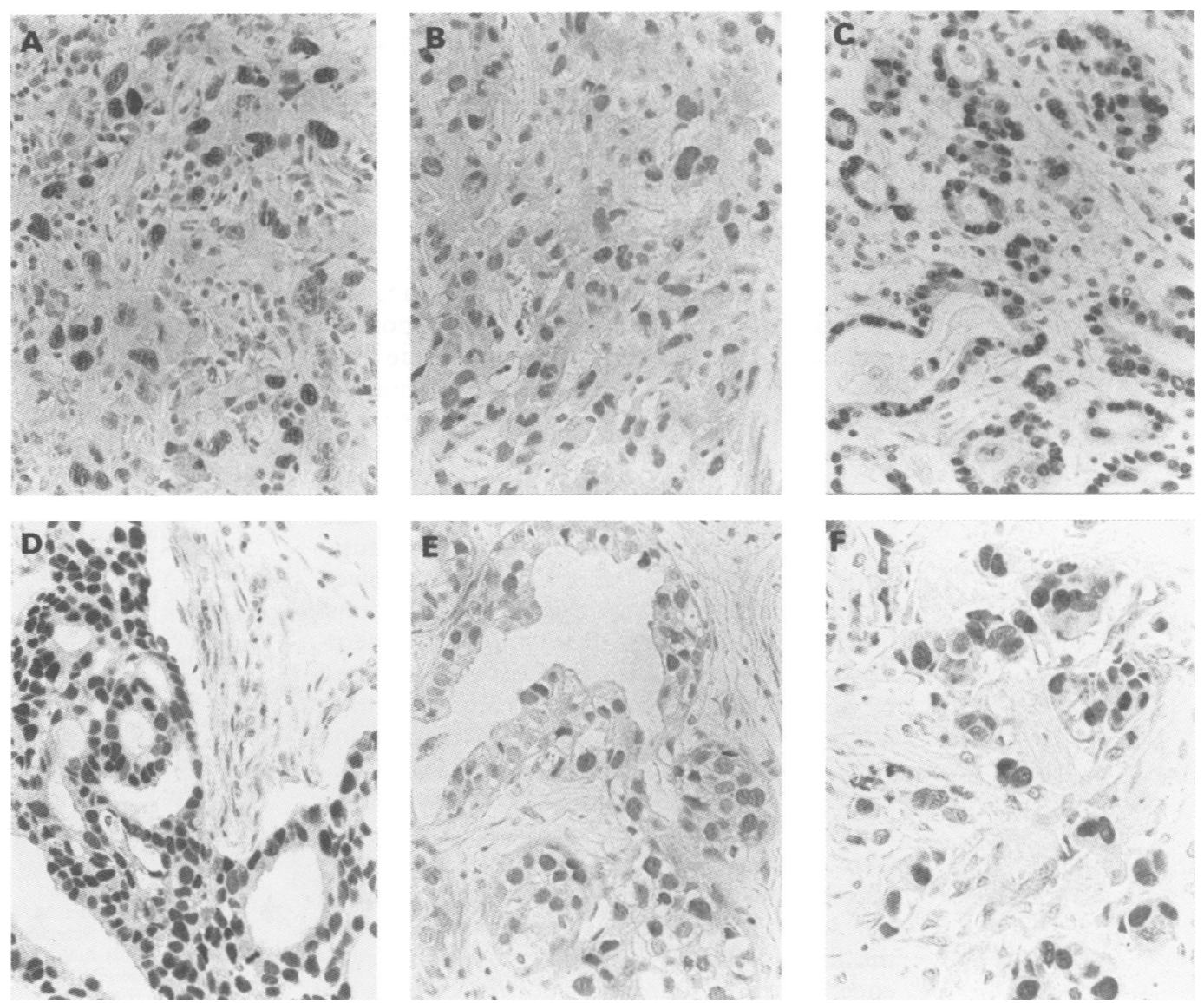

Figure 4 Positive immunohistochemical staining for $p 53$ protein in malignant tissues from six members of family 2. (A) Prostatic carcinoma from $I I \cdot 4,(B)$ ureteric carcinoma from II $\cdot 5$, (C) gastric carcinoma from II.6, (D) lymph node metastasis from primary adenocarcinoma of unknown origin from $I I I \cdot 10$, (E and $F$ ) mammary carcinomas from the proband, III $\cdot 9$, and her sister, III $\cdot 11$.

positive staining in a small number of normal epithelial cells adjacent to the tumour.

\section{Discussion}

Germline TP53 mutations have been identified as being the genetic basis of the Li-Fraumeni syndrome (LFS) ${ }^{24}$ We report the presence of TP53 mutation in one "Li-Fraumeni-like" family (family 2), but not in a family (family 1) satisfying the original criteria of $\mathrm{Li}$ and Fraumeni. ${ }^{1}$

The mutation found in family 2 was in exon 7 of the gene at position 1 in codon 245 and caused a Gly-Ser amino acid change. The same mutation was found in other affected family members and also in an unaffected person indicating that the mutation has been transmitted through the germline. This subject is young and still has a substantial risk of developing cancer. In contrast, in family 1 , no mutation was found in this region or at any other position tested within the TP53 gene. Since this study was finished further analysis of the p53 gene from family 1 has been carried out and all the exons have been analysed. No mutations were identified in exons 1-4 which were not analysed in this study ( $R$ A Eeles, personal communication). This and other studies suggest that not all LFS families have detectable germline mutations. For example, Santibanez-Koref et $\mathrm{al}^{7}$ have reported germline mutations in only two out of eight LFS families.
Originally in that study only the conserved region in exon 7 was sequenced; however a further three germline mutations have since been found by these authors in exons 4, 5, and $6 .^{9}$

The presence of positive p53 staining in malignant tissues is often indicative of an abnormally stablised p53 protein. Initially this stablisation was taken as good evidence of a mutation within the TP53 gene. However, recent reports ${ }^{16-18}$ have shown that p53 immunopositivity is not necessarily synonymous with a mutation within the gene. In the present study we have performed immunohistochemical staining on available formalin fixed paraffin embedded tissue from our two familites. Both exhibited similar staining patterns in the malignant cells. Family 1, however, also showed positive staining in normal epithelial and mesenchymal cells. No constitutional mutations were found in this family.

We have described previously a similar finding in a cancer family patient where abnormal expression of wild type p53 protein was detected in normal cells, particularly in skin surrounding the tumour. Despite extensive sequencing no mutations were identified and biochemical analysis of the p53 protein extracted from cultured normal fibroblasts indicated that the protein was wild type. ${ }^{16}$ These authors have also recently shown positive staining in keratinocytes in skin overlying benign hair follicle tumours. Although the staining 
pattern is similar to our own the degree of staining is not nearly as extensive as that observed in our families. The patient described by Eeles $e t a l^{19}$ was found to have a mutation in exon 8 of the TP53 gene whereas members from our family 1 and our previously described patient ${ }^{16}$ did not show any germline TP53 mutations. In contrast, in family 2 , we have found a germline mutation in six members of a family classified as "Li-Fraumeni-like". No positive staining in p53 was seen in normal tissue from five of the relatives; in the sixth a small number of normal epithelial cells near the tumour were weakly positive.

A mutation at codon 245 has been described previously in other Li-Fraumeni families ${ }^{24}$ and Harris $^{20}$ reviewed the frequency of p53 mutations in tumours and germline which showed that this region is a hot spot for mutations. Normal skin fibroblasts from four family members of one of their LFS families were analysed by Srivastava $e t ~ a l^{4}$ for the presence of mutant TP53. All four had the same point mutation at the second position in codon 245 (GGCGAC) causing a Gly-Asp amino acid change. Low levels of mutant and wild type $\mathrm{p} 53$ protein were detected in NSF cells from the family members carrying the germline mutation. In our study, those family members who carried the TP53 codon 245 mutation showed no evidence of strong staining of normal cells as detected immunohistochemically by the antibodies $\mathrm{CM}-1$ and DO1, although some weak staining of normal tissue was seen in the proband. These observations perhaps suggest different functional implications of the types of TP53 mutations inherited. However, further cases need to be identified and analysed in order to address this question.

It is reasonable to assume that the alteration of the TP53 gene leading to detectable levels of $\mathrm{p} 53$ protein in family 2 is because of the mutation at codon 245. A number of possibilities exist that could account for the positive immunostaining observed in family 1 , including a mutation elsewhere in the TP53 gene or a mutation in a regulator element. When the mechanism is fully elucidated it should then be possible to explain and perhaps to correlate the different types of staining patterns observed. However, irrespective of the underlying causes, it is obvious that immunostaining is not a satisfactory predictive marker of all mutations although it does show alterations in the stability of $\mathrm{p} 53$ protein which may reflect altered function.

These findings have important implications for predictive testing. It is clear that immunohistochemical staining of tumours is sufficient to identify germline mutations of TP53. Also the clinical definition used to identify Li-Fraumeni families is not sufficient to identify all patients with TP53 mutations.

We are grateful to Dr M A T Williams, ICRF Genetic Epidemiology Laboratory, Leeds, for obtaining blood samples from all of the subjects. We are grateful to Dr R A Eeles for supplying us with unpublished information on the sequencing of the p53 gene in family 1 . We would also like to acknowledge Mrs A Mills for maintaining the family records and preparing the pedigree figures.

$1 \mathrm{Li} \mathrm{FP,} \mathrm{Fraumeni} \mathrm{JFJ,} \mathrm{Mulvihill} \mathrm{JJ,} \mathrm{et} \mathrm{al.} \mathrm{A} \mathrm{cancer} \mathrm{family}$ syndrome in twenty four kindreds. Cancer Res 1988;48: 5358-62.

2 Malkin D, Li FP, Strong LC, et al. Germline p53 mutations in a familial syndrome of breast cancer, sarcomas and in a familial syndrome of breast cancer,

3 Levine AJ, Momand J, Finlay CA. The p 53 tumour suppressor gene. Nature 1991;351:453-6.

4 Srivastava S, Zou Z, Pirollo K, Blattner W, Chang EH. Germline transmission of a mutated 53 gene in a cancerprone family with Li-Fraumeni syndrome. Nature 1990 348:747-9.

5 Law JC, Strong LC, Chidambaram A, Farrell RE. A germline mutation on exon 5 of the p 53 gene in an extended cancer family. Cancer Res 1991;5:6385-7.

6 Metzger AK, Sheffield VC, Duyk G, Daneshaur L, Edwards MSB, Cogen PH. Identification of a germline mutation in the $\mathrm{p} 53$ gene in a patient with an intracranial ependymoma Proc Natl Acad Sci USA 1991;88:7825-9.

7 Santibanez-Koref MF, Birch JM, Hartley AL, et al. p53 germline mutation in Li-Fraumeni syndrome. Lancet 1991;338:1490-1.

8 Brugieres L, Gardes M, Moutou C, Chompret A, Meresse V, Martin A. Screening for germline p53 mutations in children with malignant-tumors and a family history of children with malignant-tumors and

9 Birch JM, Hartley AL, Tricker KJ, et al. Prevalence and diversity of constitutional mutations in the p53 gen among 21 Li-Fraumeni families. Cancer Res 1994;54: 1298-304

10 Easton DF, Bishop DT, Ford D, Crockford GP. Geneticlinkage analysis in familial breast and ovarian cancer. $A m$ $\Im$ Hum Genet 1993;52:678-701.

11 Prosser J, Porter D, Coles C, et al. Constitutional p53 mutation in a non-Li-Fraumeni cancer family. $\mathrm{Br} 7 \mathrm{Cance}$ 1992;65:527-8.

12 Sidransky D, Tokino T, Helzlsouer K, et al. Inherited p53 gene mutations in breast cancer. Cancer Res 1992.52: 2984-6.

13 MacGeoch C, Kennedy S, Spurr NK. Strategies for amplification of human TP53 gene sequences. Technique 1992;3:179-83.

14 Voitesek B, Bartek J, Midgley CA, Lane DP. An immunochemical analysis of the human nuclear phosphoprotein-p53-new monoclonal-antibodies and epitope phoprotein-p53-new monocional-antibodies and epitope mapping using

15 Midgley CA, Fisher CJ, Bartek J, Vojtesek B, Lane D, Barnes DM. Analysis of p53 expression in human tumours - an antibody raised against human p53 expressed in Escherichia-coli. f Cell Sci 1992;101:183-9.

6 Barnes DM, Hanby AM, Gillett CE, et al. Abnormal expression of wild type-p 53 protein in normal cells of a cancer family patient. Lancet 1992;340:259-63.

17 MacGeoch C, Barnes D, Newton JA, et al. p53 protein detected by immunohistochemical staining is not always mutant. Disease Markers 1993;11:239-50.

18 Coles C, Condie A, Chetty U, Steel CM, Evans HJ, Prosser J53 mutations in breast cancer. Cancer Res 1992;52: 5291-8.

19 Eeles RA, Warren W, Knee G, et al. Constitutional mutation in exon 8 of the p53 gene in a patient with multiple primary tumours: molecular and immunohistochemical primary tumours: molecular and im

20 Harris CC 53 . at the crossroads of molecular carcinogenesis and risk assessment. Science 1993;262:1980-1. 